\title{
The Operational Experience, Challenges and Performance of the ATLAS Semiconductor Tracker during LHC Run 2
}

\author{
Shigeki Hirose*, on behalf of the ATLAS SCT Group \\ Albert-Ludwigs-Universität Freiburg, Germany \\ E-mail: shigeki.hirose@physik.uni-freiburg.de
}

The Large Hadron Collider completed its Run 2 operation period from 2015 through 2018, which delivered an integrated luminosity of $156 \mathrm{fb}^{-1}$ at a centre-of-mass $p p$ collision energy of $13 \mathrm{TeV}$. This marked ten years of successful operation of the ATLAS Semiconductor Tracker (SCT), which operated during Run 2 with instantaneous luminosity and pileup conditions that were far in excess of what the SCT was originally designed to meet. Nevertheless, thanks to various improvements on its operation, the SCT achieved high performance with a hit efficiency greater than 99\%. The effects of radiation damage, in particular significant increase of the leakage current and change of the full depletion voltage, were observed during Run 2. Although those may risk the SCT operation in the Run 3 operation period starting in 2021, studies reveal that a sufficient margin of the SCT specification remains, which will ensure the safe operation until 2024.

The 28th International Workshop on Vertex Detectors - Vertex2019

13-18 October, 2019

Lopud, Croatia

${ }^{*}$ Speaker. 


\section{Introduction}

The ATLAS detector [1] is a multi-purpose detector composed of several sub-detector systems: the inner detector, the calorimeter and the muon spectrometer. It targets high- $p_{\mathrm{T}}$ objects originating from massive particles, such as the Higgs boson, the $t$ quark and undiscovered hypothetical particles such as supersymmetric particles, produced in $p p$ collisions provided by the Large Hadron Collider (LHC). The LHC was operated at the centre-of-mass energy of 7-8 TeV in the Run 1 operation (2010-2012) with an instantaneous luminosity of up to $8 \times 10^{33} / \mathrm{cm}^{2} / \mathrm{s}$ and parameters were increased to $13 \mathrm{TeV}$ with up to $2 \times 10^{34} / \mathrm{cm}^{2} / \mathrm{s}$ in Run 2 (2015-18).

The semiconductor tracker (SCT) is the second innermost tracking system of the ATLAS detector composed of silicon strip sensors [2]. The system is overviewed in Ref [3] while only several important aspects are described below. A sensor consists of $768 p^{+}$-strips on an $n$-bulk, and the rear-side electrode comprising of an aluminised $n^{+}$silicon layer. An SCT module has two sensors on each of the two sides with a stereo angle of $40 \mathrm{mrad}$. The small angle allows to have a tracking resolution in the direction along the strips. Typically a high-voltage (HV) of $150 \mathrm{~V}$ is applied across the sensor. Twelve ABCD3TA chips [4] are placed on the module to process signals. In total, 2112 modules are mounted on the four barrel layers (barrel 3 to 6) of the SCT cylinder [5] while 1976 modules compose nine disks (disk 1 to 9 ) on each endcap [6]. The endcap in the positive $z$-direction (the $z$-axis is defined as the direction of the beam circulating counter-clockwise) is called "endcap A" while the opposite is "endcap C".

The ABCD ASIC amplifies, shapes and discriminates the charge collected on each strip to form a binary output corresponding to a threshold of $1 \mathrm{fC}$. Data are sent via two optical links (one for each side of the module) to a readout driver (ROD) via back of crate (BOC) card. Each ROD/BOC pair processes data from up to 48 modules, and sends the formatted data to the ATLAS central data acquisition (DAQ) system.

The SCT has been operated for nearly ten years since the beginning of Run 1. Ageing and radiation damage effects gradually affect the SCT sensors and the DAQ system. In the following sections, performance of the SCT and radiation damage on the sensor observed during the LHC Run 2 operation are discussed.

\section{SCT operation in LHC Run 2}

During the Run 2 operation period, the LHC delivered an integrated luminosity of $156 \mathrm{fb}^{-1}$ to the ATLAS experiment. This was significantly larger than that in Run $1\left(28.3 \mathrm{fb}^{-1}\right)$. The abundant data statistics enabled ATLAS to perform diverse physics programs. On the other hand, it was a challenging period for the SCT because the instantaneous luminosity and pileup conditions were nearly a factor of two higher than the original design values. The severe conditions increased the total radiation dose as well as operational issue such as data bandwidth limitation and increased rates of single event upsets (SEUs). Nevertheless the SCT maintained its high performance: $0.15 \%$ of the system dead time and $99.7 \%$ of the data-quality efficiency [7]. As of the end of $2018,98.6 \%$ of the detector elements were active.

The successful SCT operation was based on various improvements on the DAQ system. To handle the higher data transmission rates, the number of RODs were increased from 90 to 128 

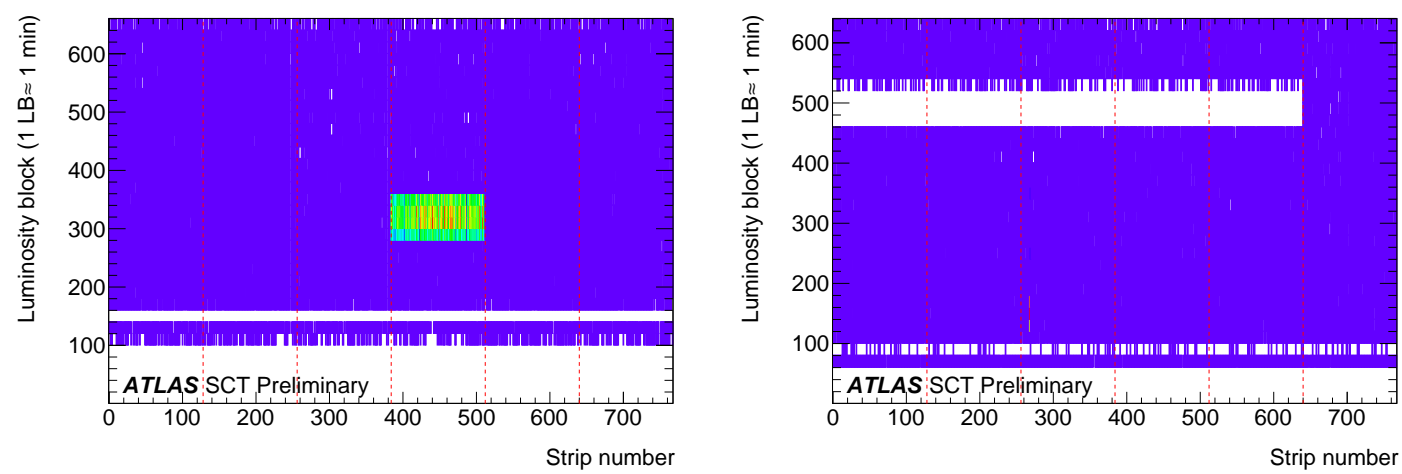

Figure 1: Noise hit map from two sensors containing (left) a noisy chip and (right) five consecutive quiet chips [8]. The noise hit is measured using empty bunch crossings during physics data-taking. The vertical axis shows a luminosity block (LB), where $1 \mathrm{LB}$ corresponds to one minute. A set of 128 strips belongs to one chip.

before the start of Run 2. This enabled the maximum number of modules per ROD to be reduced to 36 so that the higher occupancies and trigger rates could be accommodated within the existing bandwidth limitation. Furthermore, a more aggressive on-chip data compression scheme was deployed. During Run 1 operation, data were read out from three consecutive time bins centred on the triggered bin (a time bin has a time window of $25 \mathrm{~ns}$, corresponding to the frequency of bunch crossings). From Run 2, only the hit information on a triggered bin was read out while hits on the neighbour time bins were used for decision-making to send data from the strip. Typically, no hit was required in the preceding bunch in order to veto signals from the preceding collision (this is often referred to as " $01 \mathrm{X}$ " pattern). In addition, the data size was reduced by rejecting all metadata except the hit information. The improved data transmission scheme achieved to retain the sufficient bandwidth to handle the data up to $\sim 60$ collisions per event $(\mu)$, which was the maximum $\mu$ during Run 2.

The high-rate radiation increased a probability of causing an SEU on a configuration bit. If the bit flip occurred on the threshold configuration register, the chip may become either noisy or quiet (Figure 1 (left)). An SEU may also erroneously flag a chip on the last chip in the chain, which interrupted data readout from several consecutive chips (Figure 1 (right)). These problems caused low hit efficiency due to noisy strips masked, or loss of hits. The problems were effectively cleared by introducing a global reconfiguration (GR). The GR reconfigured all modules every 90 minutes at the cost of about one second of dead time. The data loss due to the dead time was negligibly small.

\section{SCT performance}

\subsection{Hit efficiency}

The hit efficiency is one of the key parameters of the SCT performance. It is defined as the probability of finding a hit created by the charged particle passing through the silicon sensor. If the particle traverses a disabled chip, a disabled module or a dead region of the module, it is ignored 


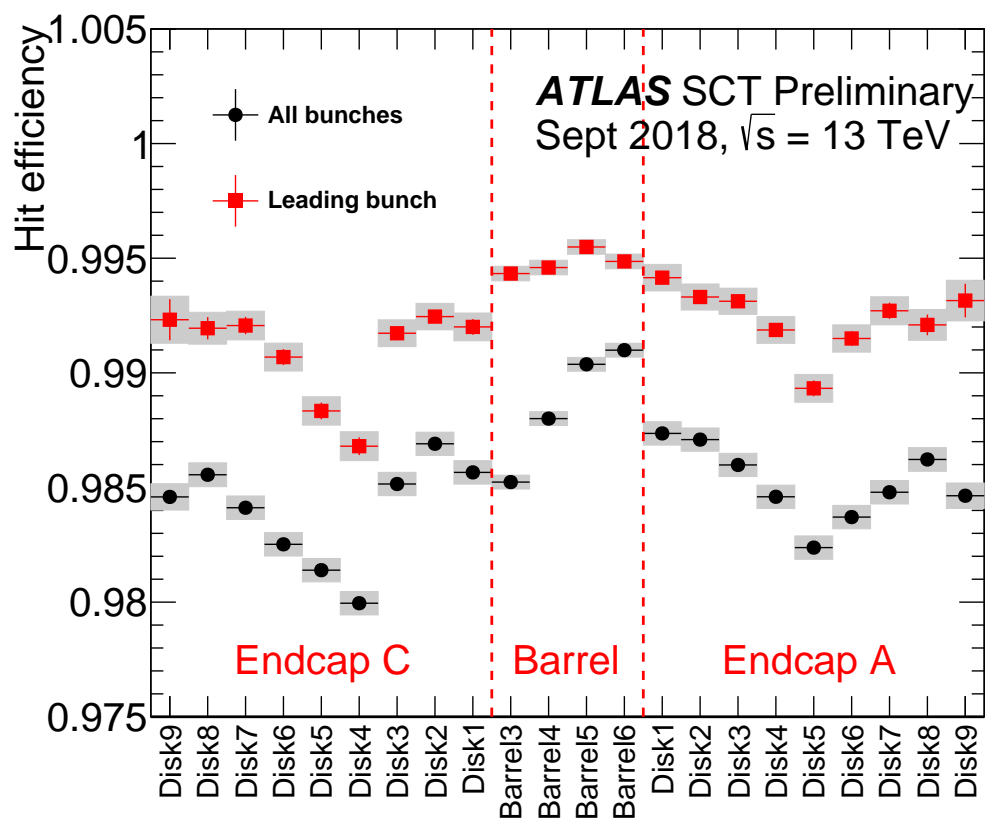

Figure 2: Average hit efficiency on each barrel layer and endcap disk [8]. The red dots show the hit efficiency measured using only the leading bunch of bunch trains, while the black dots are from the measurement using all bunch crossings. Systematic uncertainties estimated in Ref. [3] are shown by the grey bands.

from the hit efficiency calculation; however, an isolated masked or disabled strip may cause an inefficiency.

Figure 2 shows the hit efficiency of each barrel layer and endcap disk measured using a typical $p p$ collision run in September 2018. The intrinsic hit efficiency can be measured using data from crossings of a leading bunch in the bunch train so there is no signal from the preceding bunch crossing. Overall, the hit efficiency is as high as $99 \%$. This is not only the time for this particular run, but the high hit efficiency was achieved over the entire Run 2 operation.

If all bunches are used, the hit efficiency is estimated to be lower by $\sim 1 \%$. This is due to the 01X requirement discussed in Sec. 2. While this requirement effectively rejects hits related to the previous collision, a small fraction of the actual hits are rejected in case two different particles hit the same strip in two consecutive bunch crossings. A typical hit occupancy in Run 2 was $\sim 1 \%$, hence, roughly $1 \%$ of actual hits were discarded. Thanks to redundancy of having multiple layers, the impact of the $1 \%$ loss of the hit efficiency is negligibly small on the track finding efficiency.

Figure 2 indicates that the hit efficiency is significantly different among the layers and disks. The difference is induced by masked or disabled strips. Those include strips exceeding the noise occupancy of $1.5 \%$ and disabled by the online detector calibration procedure. During physics datataking, the number of noisy strips constantly increased. To maintain the hit efficiency, it was hence essential to perform a threshold calibration as frequently as possible and reset the threshold. The online calibration became more important in the later years of Run 2, where the radiation damage was more significant. 


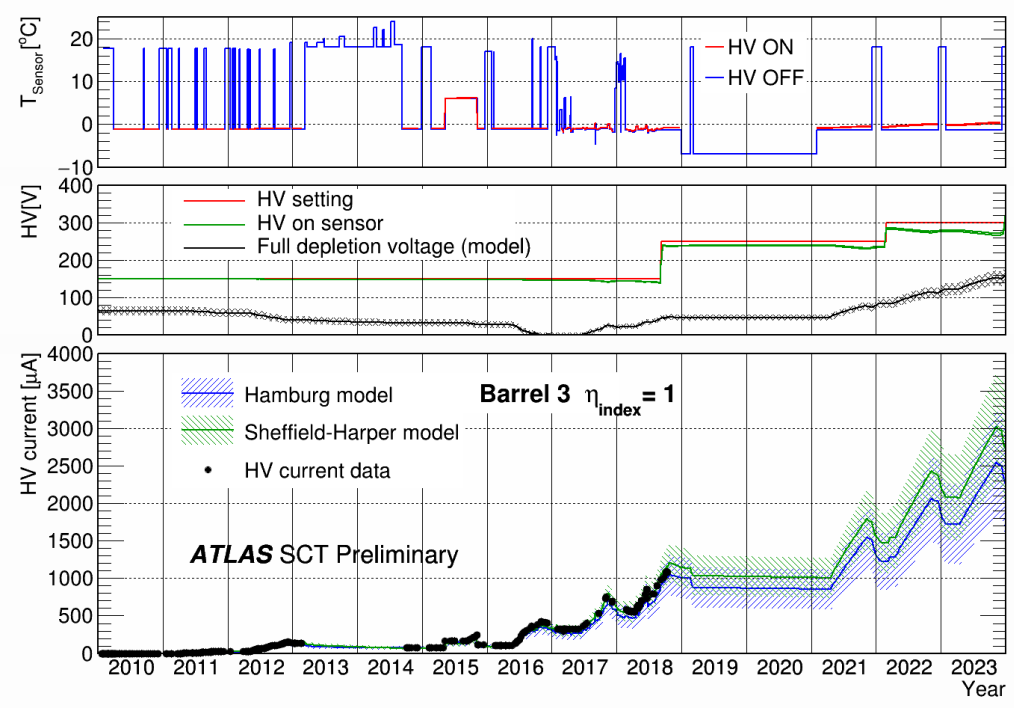

Figure 3: Evolution of the leakage current, shown in the bottom panel, over the SCT operation period since 2010 [9]. The dots are measured values while the coloured lines with bands show model predictions based on a FLUKA simulation study and two different radiation damage models. The top and middle panels show histories of the sensor temperature and the HV, respectively.

\subsection{Radiation damage}

While the hit efficiency was stable and high throughout Run 2, effects from radiation damage became gradually more significant. For example, a hit efficiency on the innermost barrel layer decreased by about $1 \%$ in the middle of 2018. Since it was not related to an increase of the masked or disabled strips, the $\mathrm{HV}$ on the innermost layer was raised from $150 \mathrm{~V}$ to $250 \mathrm{~V}$. This was the first case that the operational HV was changed in the past ten-year operation of the SCT. Although its impact on data quality was marginal, such radiation damage effects could be more important in the LHC Run 3 operation starting in 2021 until 2024.

\subsubsection{Leakage current}

The leakage current is a good indicator of the exposed radiation flux because it increases as a function of the total radiation dose. It has been monitored since the beginning of Run 1 (20102012) as shown in Figure 3 together with model predictions based on the Hamburg model [10] and the Sheffield model [11]. Since the leakage current significantly depends on the sensor temperature and $\mathrm{HV}$ as well as a profile of the integrated luminosity, those histories were tracked and compared to the predictions. The measured values agree well with the predictions within the uncertainty, indicating a good understanding of the radiation damage. In this figure, the prediction is projected to the second LHC shutdown (LS2; from 2019-2020) and the Run 3 operation period.

\subsubsection{Full depletion voltage}

Estimation of the full depletion voltage $V_{\mathrm{FD}}$ is more complicated than that of the leakage current. Since it is not possible to measure capacitance directly on the SCT sensor, $V_{\mathrm{FD}}$ needs to be inferred from measurements of various relevant parameters. 


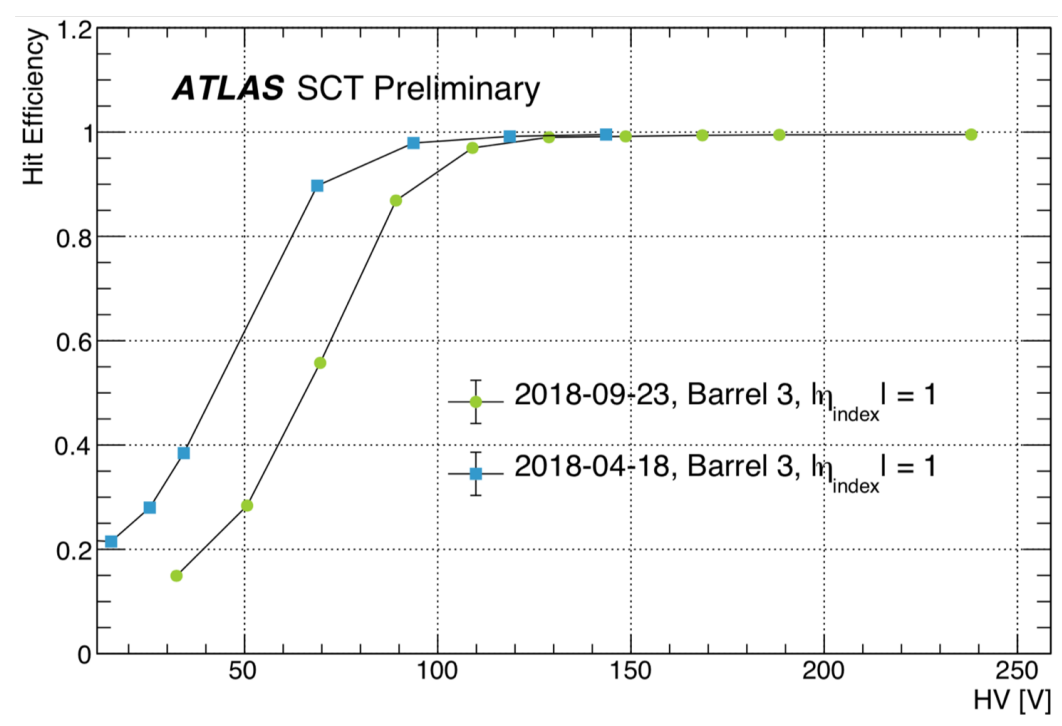

Figure 4: Hit efficiency as a function of the HV [9]. Two measurements in the beginning and the end of the 2018 run are compared.

In the case of a $p^{+}$-in- $n$ sensor, the depletion zone grows from the junction between the $p^{+}-$ strip and the $n$-bulk. After type-inversion where the $n$-bulk is converted to $p$-bulk due to increase of silicon lattice defects, the depletion zone is formed from the backside electrode. Therefore the HV dependence of the hit efficiency, as shown in Figure 4, becomes more sensitive to $V_{\mathrm{FD}}$; it sharply drops when the $\mathrm{HV}$ is below $V_{\mathrm{FD}}$. From this figure, $V_{\mathrm{FD}}$ at the end of Run 2 is estimated to be around $100 \mathrm{~V}$, with an increase of about $20 \mathrm{~V}$ during the 2018 run.

The HV dependence of the leakage current and the noise also indicate $V_{\mathrm{FD}}$ (Figure 5). The latter is shown as an equivalent noise charge (ENC), and estimated from a hit occupancy measurement by scanning the threshold setting for a fixed input charge [12]. For both leakage current and noise, the transition point to the plateau roughly indicates $V_{\mathrm{FD}}$. These figures suggest that $V_{\mathrm{FD}}$ is currently around $80-120 \mathrm{~V}$ for barrel 3; however, the transition is not perfectly clear, so the actual value of $V_{\mathrm{FD}}$ is ambiguous. If $V_{\mathrm{FD}}$ from different layers is relatively compared, both measurements indicate that the highest value of $V_{\mathrm{FD}}$ is on barrel 3 and lowest is on barrel 6. This is as expected because the innermost layer is exposed to the highest amount of radiation.

In Figure 5 (right), there is a bump in the ENC around 80-100 V, which is called the "knee". As shown in Figure 6, the knee on barrel 3 appeared in 2017. It is expected to be correlated with the onset of full depletion after the type inversion, although the exact behaviour is not yet understood in the absence of the modelling of the evolution of inter-strip capacitance and backplane capacitance with $\mathrm{HV}$ following radiation damage. Using this feature, it is confirmed that all SCT modules were type-inverted until 2018. From the knee voltage, the beneficial annealing is noticeable during LS2 in 2019.

In summary, all measurements indicate that $V_{\mathrm{FD}}$ is around $100 \mathrm{~V}$ at the end of Run 2. Although the uncertainty is large, it seems to be significantly higher than $50 \mathrm{~V}$ from a prediction based on the Hamburg model [10]. It should be noted that the Hamburg model assumes a uniform space charge distribution while the distribution can be non-uniform after the sensor is irradiated. 

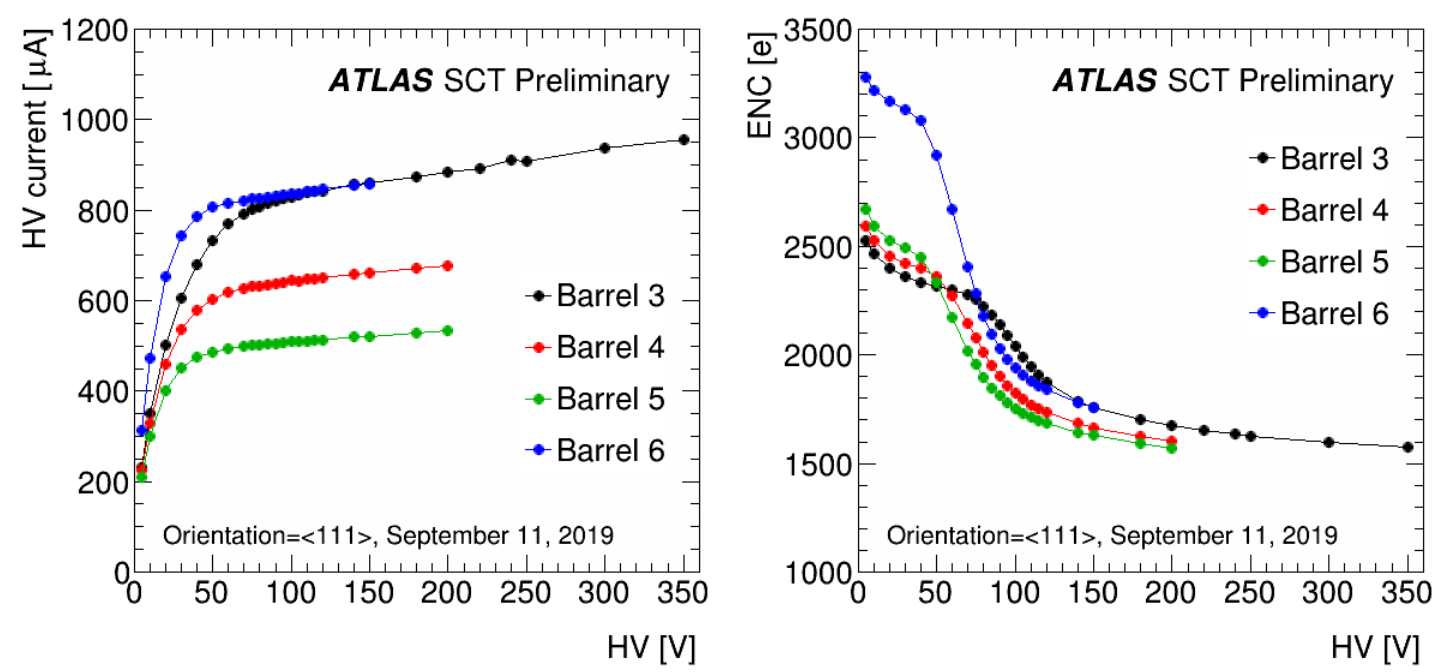

Figure 5: HV dependence of the leakage current (left) and the noise (right) measured in September 2019 [8]. In both plots, barrel 6 has the highest values although it is the outermost layer with lower radiation dose. This is due to the higher cooling temperature setting in order to protect the outer detector structure.

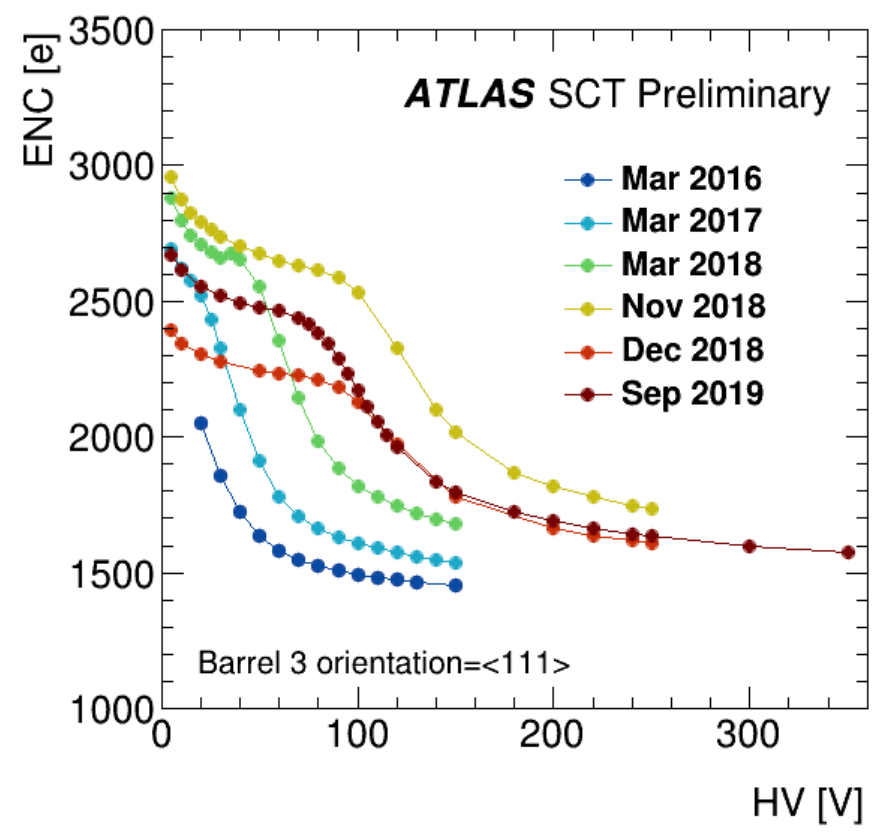

Figure 6: HV dependence of the noise on barrel 3, measured from March 2016 until September 2019 [8].

\section{Prospects for Run 3}

The LHC Run 3 operation is planned from 2021 through 2024. The run conditions will be almost the same as that of Run 2: a maximum instantaneous luminosity of $2 \times 10^{34} / \mathrm{cm}^{2} / \mathrm{s}$ and an integrated luminosity of $220-230 \mathrm{fb}^{-1}$. Since the SCT system and operation procedure have been well established thanks to various improvements in the past operation periods, no major upgrade 


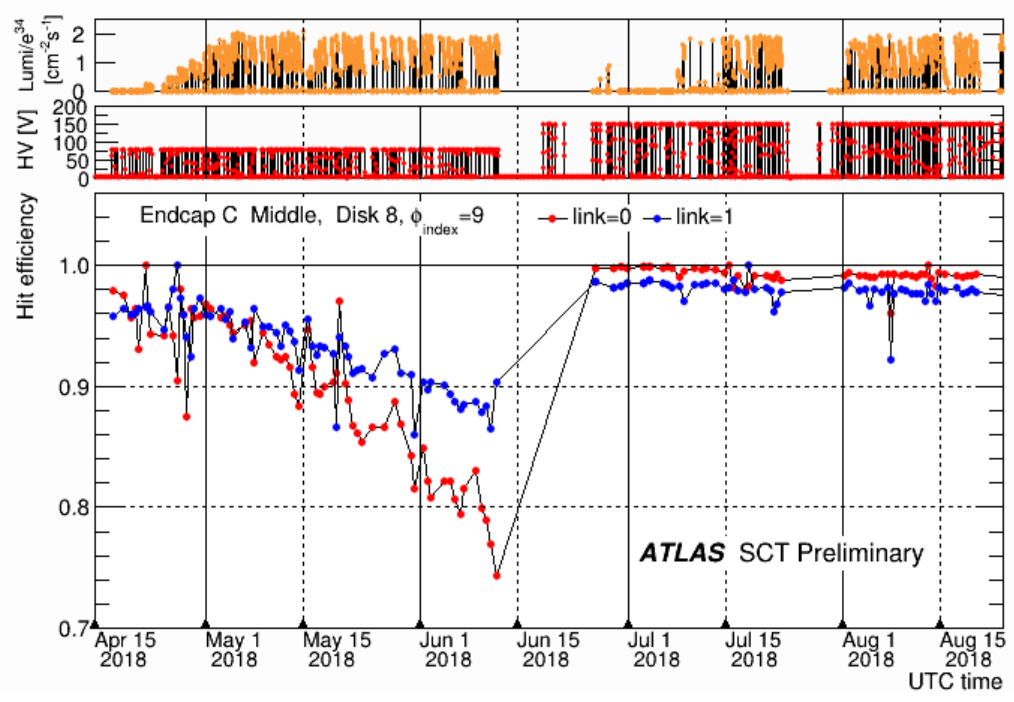

Figure 7: Evolution of the hit efficiency of a module located on disk 8 of endcap C [8]. The top two panels show the instantaneous luminosity and the HV on this module, respectively. As shown in the middle panel, only $80 \mathrm{~V}$ was applied to this module. This is due to an anomalous leakage current observed in Run 1 . The low hit efficiency was recovered around 15 th June 2018 by an increase of the HV to $150 \mathrm{~V}$.

towards Run 3 is planned. However, a closer monitoring on the performance is essential because it will be gradually deteriorated due to radiation damage.

From the leakage current study in Sec. 3.2.1, a good understanding of the radiation damage on SCT sensors is confirmed. A concern which can be caused by large leakage current is a positive feedback as follows; the leakage current induces more power dissipation of the sensor, which increases the leakage current due to a higher sensor temperature. That may finally lead to a thermal runaway whereby the module cannot be operated. A simulation study based on results from the leakage current measurement and the Run 3 operation plan shows sufficient headroom even at the end of Run 3.

The Hamburg model predicts that $V_{\mathrm{FD}}$ will increase up to about $150 \mathrm{~V}$ at the end of Run 3 , which is shown in the middle panel of Figure 3. As discussed in Sec. 3.2.2, this prediction may underestimate $V_{\mathrm{FD}}$ by $\sim 50 \mathrm{~V}$. However, since all modules were qualified to sustain up to $500 \mathrm{~V}$ [2], a sufficient margin is assured.

The HV will need to be increased whenever the hit efficiency deteriorates during operation. An example of the efficiency degradation is shown in Figure 7. It should be noted that this is a module to which a lower operational HV was applied. Although this is not a module with a typical condition, this figure is a good example indicating that the hit efficiency will slowly decrease with about $10-20 \%$ per month. It is too slow to be detected by the current run-by-run basis data quality monitoring procedure. In order to monitor a long-term trend and perform a quick diagnosis on low efficiency modules once found, a quick module performance analysis tool is being developed.

\section{Conclusion}

During the LHC Run 2 operation from 2015 until 2018, the SCT was successfully operated 
with high data quality and small dead time. During this operation period, radiation damage became more significant, which required to modify the operation conditions; one example is the increase of operational HV up to $250 \mathrm{~V}$. For Run 3, no serious operational problem is foreseen; however, radiation damage may affect the SCT performance more. It is therefore essential to monitor carefully detector conditions such as leakage current and hit efficiency so that an action can be taken in case a performance deterioration is observed. Based on the improved operation scheme, the SCT will continue to provide high quality data to the ATLAS experiment in LHC Run 3.

\section{References}

[1] ATLAS Collaboration, The ATLAS Experiment at the CERN Large Hadron Collider, JINST 3, S08003 (2008).

[2] A. Ahmad et al., The silicon microstrip sensors of the ATLAS semiconductor tracker, Nucl. Instrum. Meth. A 578, 98 (2007).

[3] ATLAS Collaboration, Operation and performance of the ATLAS semiconductor tracker, JINST 9 , P08009 (2014).

[4] F. Campabadal et al., Design and performance of the ABCD3TA ASIC for readout of silicon strip detectors in the ATLAS semiconductor tracker, Nucl. Instrum. Meth. A 552, 292 (2005).

[5] A. Abdesselam et al., The barrel modules of the ATLAS semiconductor tracker, Nucl. Instrum. Meth. A 568, 642 (2006).

[6] A. Abdesselam et al., The ATLAS semiconductor tracker end-cap module, Nucl. Instrum. Meth. A $\mathbf{5 7 5}, 353$ (2007).

[7] ATLAS Collaboration, ATLAS data quality operations and performance for 2015-2018 data-taking, arXiv:1911.04632; submitted to JINST.

[8] ATLAS Collaboration, SCT-2019-002, https://atlas.web.cern.ch/Atlas/GROUPS/PHYSICS/PLOTS/SCT-2019-002/.

[9] ATLAS Collaboration, SCT-2019-001, https://atlas.web.cern.ch/Atlas/GROUPS/PHYSICS/PLOTS/SCT-2019-001/.

[10] M. Moll, Radiation damage in silicon particle detectors: Microscopic defects and macroscopic properties, Ph.D. Thesis, Universität Hamburg, Germany (1999).

[11] R. Harper, Radiation Damage studies of the silicon microstrip detectors and finding an intermediate mass Higgs Boson at ATLAS, Ph.D. Thesis, University of Sheffield, UK (2001).

[12] A. Abdesselam et al., The data acquisition and calibration system for the ATLAS Semiconductor Tracker, JINST 3, P01003 (2008). 